PROCEEDINGS OF THE

AMERICAN MATHEMATICAL SOCIETY

Volume 136, Number 6, June 2008, Pages 2081-2090

S 0002-9939(08)09355-6

Article electronically published on February 14, 2008

\title{
ON THE DECAY PROPERTIES OF SOLUTIONS TO A CLASS OF SCHRÖDINGER EQUATIONS
}

\author{
L. DAWSON, H. MCGAHAGAN, AND G. PONCE
}

(Communicated by Matthew J. Gursky)

\begin{abstract}
We construct a local in time, exponentially decaying solution of the one-dimensional variable coefficient Schrödinger equation by solving a nonstandard boundary value problem. A main ingredient in the proof is a new commutator estimate involving the projections $P_{ \pm}$onto the positive and negative frequencies.
\end{abstract}

\section{INTRODUCTION}

In [5], T. Kato showed that the semigroup $\left\{e^{-t \partial_{x}^{3}}: t \geq 0\right\}$ in the space $L^{2}\left(e^{2 \beta x} d x\right)$ with $\beta>0$ is formally equivalent to the semigroup $e^{-t\left(\partial_{x}-\beta\right)^{3}}$ in $L^{2}(\mathbb{R})$. Among the immediate consequences of this result is that if $u \in C\left([0, T]: H^{1}(\mathbb{R})\right)$ is a strong solution of the $k$-generalized Korteweg de Vries (KdV) equation,

$$
\partial_{t} u+\partial_{x}^{3} u+u^{k} \partial_{x} u=0, \quad k=1,2, \ldots,
$$

with data $u_{0} \in L^{2}\left(e^{2 \beta x} d x\right)$, then $u \in C\left([0, T]: L^{2}\left(e^{2 \beta x} d x\right)\right) \cap C^{\infty}(\mathbb{R} \times(0, T])$. In other words, the solution $u=u(x, t)$ satisfies the persistence property $e^{\beta x} u \in$ $C\left([0, T]: L^{2}(\mathbb{R})\right)$ and a "parabolic" regularization, $u \in C^{\infty}(\mathbb{R} \times(0, T])$.

Since results for solutions of the $k$-generalized KdV equation and Schrödinger equations of the type

$$
\text { (a) } \partial_{t} u-i \Delta u=f(|u|) u
$$

(b) $\partial_{t} u-i(\Delta u+W(x, t) u)=F(x, t)$,

run parallel - for instance, solutions of both satisfy Strichartz estimates, local smoothing effects of the Kato type, and persistence properties in $H^{s}(\mathbb{R})$, the weighted spaces $H^{s}(\mathbb{R}) \cap L^{2}\left(|x|^{k}\right)$, and the Schwartz space - one may ask what the equivalent result to that described above for the KdV equation is in the case of Schrödinger equations. One first notices that even for the free Schrödinger group $\left\{e^{i t \Delta}: t \in \mathbb{R}\right\}$, both of the above properties fail: assuming we are in $\mathbb{R}^{1}\left(\Delta=\partial_{x}^{2}\right)$ for simplicity, we can construct initial data $u_{0} \in L^{2}(\mathbb{R}) \cap L^{2}\left(e^{2 \beta x} d x\right)$ such that $e^{i t \partial_{x}^{2}} u_{0} \notin L^{2}\left(e^{2 \beta x} d x\right) \cup C^{\infty}(\mathbb{R})$ for any $t>0$.

Received by the editors March 6, 2007.

2000 Mathematics Subject Classification. Primary 35J10; Secondary 35B65.

The first author was supported by NSF grants.

The second author was supported by an NSF postdoctoral fellowship.

The third author was supported by NSF grants. 
Roughly, the difficulty lies in the fact that if $u(x, t)=e^{i t \partial_{x}^{2}} u_{0}(x)$, then $v(x, t):=$ $e^{\beta x} u(x, t)$ formally solves the equation

$$
\partial_{t} v-i\left(\partial_{x}-\beta\right)^{2} v=\partial_{t} v-i \partial_{x}^{2} v+2 i \beta \partial_{x} v-i \beta^{2} v=0,
$$

whose associated initial value problem (IVP) is ill-posed in $L^{2}(\mathbb{R})$. However, the operator $2 i \beta \partial_{x}$, whose symbol is $-2 \beta \xi$, introduces a parabolic structure in the negative frequency for positive time and in the positive frequency for negative time. Thus, to find $L^{2}$-solutions of equation (1.3) in the time interval $[0, T]$, one needs to consider a "boundary value problem" for (1.3) where

$$
\begin{aligned}
& v_{-}(x, 0)=P_{-} v(x, 0):=\left(\chi_{(-\infty, 0)}(\xi) \hat{v}(\xi, 0)\right)^{\vee}(x), \\
& v_{+}(x, T)=P_{+} v(x, T):=\left(\chi_{(0, \infty)}(\xi) \hat{v}(\xi, T)\right)^{\vee}(x)
\end{aligned}
$$

are prescribed. In this case, one finds the solution

$$
v(x, t)=e^{t\left(i \partial_{x}^{2}-2 \beta D_{x}+i \beta^{2}\right)} v_{-}(x, 0)+e^{-(T-t)\left(i \partial_{x}^{2}+2 \beta D_{x}+i \beta^{2}\right)} v_{+}(x, T),
$$

with $D_{x} h(x):=\left(-\partial_{x}^{2}\right)^{1 / 2} h(x)=(c|\xi| \hat{h}(\xi))^{\vee}(x)$. Then,

$$
\sup _{[0, T]}\|v(t)\|_{2} \leq c\left(\left\|v_{+}(x, T)\right\|_{2}+\left\|v_{-}(x, 0)\right\|_{2}\right),
$$

$c$ independent of $\beta>0$ and $T$, and $v \in C^{\infty}(\mathbb{R} \times(0, T))$. We observe that in formula (1.5), the positive and negative frequencies do not interact and, also, that $u(x, t):=$ $e^{-\beta x} v(x, t)$ is not necessarily an $L^{2}$-solution of the free Schrödinger equation.

The following estimate established in 7 of the type described in (1.6) for a linear Schrödinger equation with lower order variable coefficients (1.2) (b) was a key step in the proof of the unique continuation results obtained in [7] and [4].

Lemma ([7]). There exists $\epsilon>0$ such that if $W: \mathbb{R}^{n} \times[0, T] \rightarrow \mathbb{C}$ satisfies $\|W\|_{L_{t}^{1} L_{x}^{\infty}} \leq \epsilon$ and $u \in C\left([0, T]: L_{x}^{2}\left(\mathbb{R}^{n}\right)\right)$ is a strong solution of the equation (1.2) (b) with

$$
u_{0}=u(\cdot, 0), u_{T} \equiv u(\cdot, T) \in L^{2}\left(e^{2 \beta x_{1}} d x\right), \quad F \in L^{1}\left([0, T]: L_{x}^{2}\left(e^{2 \beta x_{1}} d x\right)\right)
$$

for some $\beta \in \mathbb{R}$, then there exists $c$ independent of $\beta$ such that

$$
\sup _{0 \leq t \leq T}\left\|e^{\beta x_{1}} u(\cdot, t)\right\|_{2} \leq c\left(\left\|e^{\beta x_{1}} u_{0}\right\|_{2}+\left\|e^{\beta x_{1}} u_{T}\right\|_{2}+\int_{0}^{T}\left\|e^{\beta x_{1}} F(\cdot, t)\right\|_{2} d t\right) .
$$

Notice that in the above result one assumes the existence of a reference solution $u(x, t)$ of equation (1.2) (b) and shows that under hypothesis (1.7), exponential decay in the time interval $[0, T]$ is preserved.

The $L^{2}$-well-posedness of the IVP associated to the equation

$$
\partial_{t} w=i \Delta w+b(x) \cdot \nabla_{x} w+f(x, t)
$$

has been extensively studied. In particular, S. Mizohata 8 gives the following necessary condition for the IVP associated to (1.8) to be well-posed in $L^{2}\left(\mathbb{R}^{n}\right)$ :

$$
\sup _{x \in \mathbb{R}^{n}, \omega \in \mathbb{S}^{n-1}, R>0}\left|\operatorname{Im} \int_{0}^{R} b(x+r \omega) \cdot \omega d r\right|<\infty .
$$

The gain of regularity of solutions to the variable coefficient Schrödinger equation

$$
\partial_{t} u-i \partial_{x_{j}}\left(a_{j k}(x) \partial_{x_{k}} u\right)+W(x) u=0
$$

as a consequence of its dispersive character and the decay assumptions on the data has also been studied in several works; see [2], 3], and the references therein. 
In this note, we shall combine the above ideas with some new commutator estimates to construct an exponentially decaying solution to the one-dimensional variable coefficient Schrödinger equation

$$
\partial_{t} u=i\left(\partial_{x}\left(a(x, t) \partial_{x} u\right)+W(x, t) u\right) .
$$

More precisely, we are interested in a solution $u \in C\left([0, T]: L^{2}(\mathbb{R}) \cap L^{2}\left(e^{2 \beta x} d x\right)\right)$.

To ensure that we construct $u \in L^{2}(\mathbb{R})$, we will need to refer to the following function $\varphi_{\beta}(x)$ : for $\beta>0$ we denote by $\varphi(x)=\varphi_{\beta}(x)$ a $C^{4}(\mathbb{R})$ function such that $\varphi(x)=1$ if $x \leq 0, \varphi(x)=e^{\beta x}$ if $x \geq 10 \beta$, and $\varphi(x)$ is strictly increasing on $(0,10 \beta)$.

Theorem 1.1. Let $a: \mathbb{R} \times \mathbb{R}^{+} \rightarrow \mathbb{R}$ be such that

$$
\begin{aligned}
& a \in C^{2}\left(\mathbb{R} \times \mathbb{R}^{+}\right) \cap L_{t}^{1}\left(\mathbb{R}^{+}: L_{x}^{\infty}(\mathbb{R})\right), \quad\langle x\rangle \partial_{x}^{j} a \in L_{t}^{1}\left(\mathbb{R}^{+}: L_{x}^{\infty}(\mathbb{R})\right), \quad j=1,2, \\
& a(x, t) \geq \lambda \geq 0, \quad \forall(x, t) \in \mathbb{R} \times \mathbb{R}^{+} .
\end{aligned}
$$

Let $W: \mathbb{R} \times \mathbb{R}^{+} \rightarrow \mathbb{C}$ be such that

$$
W \in L_{t}^{1}\left(\mathbb{R}^{+}: L_{x}^{\infty}(\mathbb{R})\right) .
$$

Then given $(f, g) \in P_{-} L^{2}(\mathbb{R}) \times P_{+} L^{2}(\mathbb{R})$, there exists $T=T\left(\beta ;\|a\|_{1} ;\|W\|_{L_{t}^{1} L_{x}^{\infty}}\right)>$ 0 such that (1.10) has a unique solution $u \in C\left([0, T]: L^{2}(\mathbb{R})\right)$ with

$$
e^{\beta x} u \in C\left([0, T]: L^{2}(\mathbb{R})\right)
$$

and with $P_{-}(\varphi(x) u(x, 0))=f(x)$ and $P_{+}(\varphi(x) u(x, T))=g(x)$.

If in addition $a, W \in C^{\infty}\left(\mathbb{R} \times \mathbb{R}^{+}\right) ; \lambda>0$ with

$$
\beta \lambda \geq c\left(\left\|\langle x\rangle \partial_{x} a\right\|_{L^{\infty}\left(\mathbb{R} \times \mathbb{R}^{+}\right)}+\left\|\langle x\rangle \partial_{x}^{2} a\right\|_{L^{\infty}\left(\mathbb{R} \times \mathbb{R}^{+}\right)}\right) ;
$$

and $\partial_{t}^{k} \partial_{x}^{j} a, \partial_{t}^{k} \partial_{x}^{j} W \in L^{\infty}\left(\mathbb{R} \times \mathbb{R}^{+}\right)$for any $k, j \in \mathbb{Z}^{+}$, then $u \in C^{\infty}(\mathbb{R} \times(0, T))$.

We use the notation $\langle x\rangle:=\left(1+|x|^{2}\right)^{1 / 2}$. Also, $\|a\|_{1}$ denotes the sum of the $L_{t}^{1} L_{x}^{\infty}$-norms described in (1.11):

$$
\|a\|_{1}:=\|a\|_{L_{t}^{1} L_{x}^{\infty}}+\sum_{j=1}^{2}\left\|\langle x\rangle \partial_{x}^{j} a\right\|_{L_{t}^{1} L_{x}^{\infty}} .
$$

Under the assumptions of Theorem 1.1, we do not know if the dependence on the parameter $\beta$ of the time interval $[0, T]$ can be removed as was done in [7. Also, here we shall restrict ourselves to the one-dimensional case.

To prove Theorem [1.1 we consider a system describing the time evolution of the projection of the weighted function $v:=\varphi u$ into the positive and negative frequencies. Since our equation has variable coefficients, this becomes a coupled system. It will be essential in our arguments that the coupled terms are, roughly speaking, of "order zero". We will show this using some commutator estimates such as the following one: for any $p \in(1, \infty), l, m \in \mathbb{Z}^{+} \cup\{0\}$ there exists $c=$ $c(p ; l ; m)>0$ such that

$$
\left\|\partial_{x}^{l}\left[P_{+} ; a\right] \partial_{x}^{m} f\right\|_{p} \leq c\left\|\partial_{x}^{l+m} a\right\|_{\infty}\|f\|_{p} .
$$

Clearly, the inequality (1.13) holds with $P_{-}$or $H$, the Hilbert transform, in place of $P_{+}$. In the case $l+m=1,(1.13)$ is Calderón's first commutator estimate [1]. A related version of estimate (1.13) was obtained in 9 for general positive derivatives, but did not involve the $L^{\infty}$-norm. 


\section{Proof of Theorem 1.1}

Consider the equation

$$
\partial_{t} u=i\left(\partial_{x}\left(a(x, t) \partial_{x} u\right)+W(x, t) u\right) .
$$

We wish to construct a solution $u \in L^{2}\left(\left(1+e^{2 \beta x}\right) d x\right)$ for a fixed $\beta>0$. Recall the definition of the function $\varphi(x)=\varphi_{\beta}(x)$, and define $\phi(x):=\varphi^{\prime}(x) / \varphi(x)$. Notice that $\phi(x)=\beta \chi_{\mathbb{R}^{+}}(x)$, except on the interval $0<x<10 \beta$, and that $\|\phi\|_{\infty}=\beta$.

Let $v(x, t):=\varphi(x) u(x, t)$. Then, multiplying (2.1) by $\varphi(x)$ and using the fact that $\left[\varphi ; \partial_{x}\right]=-\phi \varphi$, we have that

$$
\begin{aligned}
\partial_{t} v & =i\left(\left(\partial_{x}-\phi(x)\right)\left(a(x, t)\left(\partial_{x}-\phi(x)\right) v\right)+W(x, t) v\right) \\
& =i \partial_{x}\left(a \partial_{x} v\right)-2 i a \phi \partial_{x} v+i\left(\left(\phi^{2}-\partial_{x} \phi\right) a-\phi \partial_{x} a\right) v+i W v
\end{aligned}
$$

We will construct a solution $v \in L^{2}(\mathbb{R})$ of (2.2). This suffices since the definition of $\varphi$ then guarantees that $u$ defined by $u(x)=v(x)$ on $x \leq 0$ and $u(x)=\varphi^{-1}(x) v(x)$ on $x>0$ will be in $L^{2}\left(\left(1+e^{2 \beta x}\right) d x\right)$, and $u$ will solve (2.1).

Applying the projection operators $\left(P_{ \pm}\right)^{2}$ to equation (2.2),

$$
\begin{aligned}
\partial_{t} v_{ \pm} & =P_{ \pm}\left(i \partial_{x}\left(a \partial_{x} v_{ \pm}\right)-2 i \phi a \partial_{x} v_{ \pm}+P_{ \pm}\left(i\left(\left(\phi^{2}-\partial_{x} \phi\right) a-\phi \partial_{x} a\right)\left(v_{+}+v_{-}\right)\right)\right. \\
& \left.+P_{ \pm}\left(i W\left(v_{+}+v_{-}\right)\right)+i \partial_{x}\left[P_{ \pm} ; a\right] \partial_{x}\left(v_{+}+v_{-}\right)-2 i\left[P_{ \pm} ; a \phi\right] \partial_{x}\left(v_{+}+v_{-}\right)\right)
\end{aligned}
$$

Instead of solving (2.2) directly, we therefore consider the following coupled system:

$$
\begin{aligned}
& \partial_{t} v_{+}=P_{+}\left(i \partial_{x}\left(a \partial_{x} v_{+}\right)-2 i a \phi \partial_{x} v_{+}\right)+\Lambda_{+}\left(v_{+}, v_{-}\right), \\
& \partial_{t} v_{-}=P_{-}\left(i \partial_{x}\left(a \partial_{x} v_{-}\right)-2 i a \phi \partial_{x} v_{-}\right)+\Lambda_{-}\left(v_{+}, v_{-}\right),
\end{aligned}
$$

where

$$
\begin{aligned}
\Lambda_{ \pm}\left(v_{+}, v_{-}\right):= & P_{ \pm}\left(i\left(\left(\phi^{2}-\partial_{x} \phi\right) a-\phi \partial_{x} a\right)\left(v_{+}+v_{-}\right)+i W\left(v_{+}+v_{-}\right)\right. \\
& \left.+i \partial_{x}\left[P_{ \pm} ; a\right] \partial_{x}\left(v_{+}+v_{-}\right)-2 i\left[P_{ \pm} ; a \phi\right] \partial_{x}\left(v_{+}+v_{-}\right)\right)
\end{aligned}
$$

with data $\left(v_{+}(T), v_{-}(0)\right) \in P_{+} L^{2} \times P_{-} L^{2}$. Since all of the terms on the right-hand sides of the equations in the system (2.2) are projected, once we have established the existence and uniqueness of $L^{2}$ solutions of this system, it will follow that $P_{+} v_{+}=v_{+}$and $P_{-} v_{-}=v_{-}$, and $v:=v_{+}+v_{-}$will be the desired solution of (2.2).

Taking the $L^{2}$ norm of $\Lambda_{ \pm}$and applying Lemma 3.1. $\Lambda_{ \pm}$can be written as a sum of linear operators in $\left(v_{+}, v_{-}\right)$of "order zero":

$$
\begin{aligned}
\left\|\Lambda_{ \pm}\left(v_{+}, v_{-}\right)\right\|_{2} \leq c( & \left\|\left(\phi^{2}-\partial_{x} \phi\right) a-\phi \partial_{x} a\right\|_{\infty}+\|W\|_{\infty}+\left\|\partial_{x}^{2} a\right\|_{\infty} \\
& \left.+\left\|\partial_{x}(a \phi)\right\|_{\infty}\right)\left\|v_{+}+v_{-}\right\|_{2} \leq K(t)\left(\left\|v_{+}\right\|_{2}+\left\|v_{-}\right\|_{2}\right)
\end{aligned}
$$

with

$$
K(t):=c\left(\sum_{j=0}^{2} \beta^{j}\left\|\partial_{x}^{2-j} a(t)\right\|_{\infty}+\|a(t)\|_{\infty}+\|W(t)\|_{\infty}\right) .
$$

To prove the existence of a solution $\left(v_{+}, v_{-}\right) \in L^{2} \times L^{2}$ to (2.3) with data $\left(v_{+}(T), v_{-}(0)\right) \in P_{+} L^{2} \times P_{-} L^{2}$, we shall establish a priori estimates and local existence for a related uncoupled system, and then find $\left(v_{+}, v_{-}\right)$as a limit of these solutions.

First, we fix the time interval on which we will solve the equation. Define

$$
c_{a, \beta}(t):=c\left(\|a(t)\|_{\infty}+(1+\beta)\left\|\langle x\rangle \partial_{x} a(t)\right\|_{\infty}+\beta\left\|\langle x\rangle \partial_{x}^{2} a(t)\right\|_{\infty}\right),
$$


and let $T=T\left(\beta ;\|a\|_{1} ;\|W\|_{L_{t}^{1} L_{x}^{\infty}}\right)>0$ be such that

$$
e^{4 \int_{0}^{T} c_{a, \beta}(t) d t} \leq 2 \quad \text { and } \quad \int_{0}^{T} K(t) d t \leq 1 / 48
$$

These inequalities must hold for some $T>0$ by hypotheses (1.11) and (1.12). Also, for $v:=v_{+}+v_{-}$we define the norm

$$
\|v\|_{T}:=\sup _{[0, T]}\left\|v_{+}(t)\right\|_{2}+\sup _{[0, T]}\left\|v_{-}(t)\right\|_{2},
$$

and letting $\delta:=\left\|v_{+}(T)\right\|_{2}+\left\|v_{-}(0)\right\|_{2}$, we define the space

$$
\mathrm{X}_{T}:=\left\{v: \mathbb{R} \times[0, T] \rightarrow \mathbb{C}:\|v\|_{T} \leq 8 \delta\right\} .
$$

Next, using standard energy estimates, we obtain a priori bounds for the solutions of both of the following (uncoupled) equations on $\mathbb{R} \times[0, T]$ :

$$
\begin{aligned}
& \partial_{t} v_{+}=P_{+}\left(i \partial_{x}\left(a \partial_{x} v_{+}\right)-2 i a \phi \partial_{x} v_{+}\right)+F_{+}(x, t), \\
& \partial_{t} v_{-}=P_{-}\left(i \partial_{x}\left(a \partial_{x} v_{-}\right)-2 i a \phi \partial_{x} v_{-}\right)+F_{-}(x, t),
\end{aligned}
$$

with functions $F_{ \pm} \in L_{t}^{1}\left(\mathbb{R}^{+}: P_{ \pm} L^{2}(\mathbb{R})\right)$. Multiplying (2.8) by $\overline{v_{-}}$, integrating in the $x$-variable, and taking the real part, we obtain, after combining the identities

$$
\begin{aligned}
& \int P_{-}\left(i \partial_{x}\left(a \partial_{x} v_{-}\right)\right) \overline{v_{-}} d x=-i \int a \partial_{x} P_{-} v_{-} \overline{\partial_{x} P_{-} v_{-}} d x+i \int \partial_{x}\left[P_{-} ; a\right] \partial_{x} v_{-} \overline{P_{-} v_{-}} d x \\
& -i \int P_{-}\left(a \phi \partial_{x} v_{-}\right) \overline{v_{-}} d x=-i \int a \phi \partial_{x} P_{-} v_{-} \overline{P_{-} v_{-}} d x-i \int\left[P_{-} ; a \phi\right] \partial_{x} v_{-} \overline{P_{-} v_{-}} d x \\
& =-\int a \phi\left|D_{x}^{1 / 2} P_{-} v_{-}\right|^{2} d x-i \int\left[a \phi ; D_{x}^{1 / 2}\right]\left(D_{x}^{1 / 2} P_{-} v_{-}\right) \overline{P_{-} v_{-}} d x \\
& \quad-i \int\left[P_{-} ; a \phi\right] \partial_{x} v_{-} \overline{P_{-} v_{-}} d x
\end{aligned}
$$

with some estimates involving Lemma 3.1 and Proposition 3.2 and the hypothesis on the coefficients, that

$$
\begin{aligned}
\sup _{t \in[0, T]}\left\|v_{-}(t)\right\|_{2}+2( & \left.\int_{0}^{T} \int a(x, t) \phi(x)\left|D_{x}^{1 / 2} P_{-} v_{-}\right|^{2} d x d t\right)^{1 / 2} \\
& \leq 3\left(\left\|v_{-}(0)\right\|_{2}+\int_{0}^{T}\left\|F_{-}(t)\right\|_{2} d t\right) e^{4 \int_{0}^{T} c_{a, \beta}(\tau) d \tau} .
\end{aligned}
$$

A similar argument applied to the equation for $v_{+}(2.7)$ shows that

$$
\begin{aligned}
\sup _{t \in[0, T]}\left\|v_{+}(t)\right\|_{2}+2( & \left.\int_{0}^{T} \int a(x, t) \phi(x)\left|D_{x}^{1 / 2} P_{+} v_{+}\right|^{2} d x d t\right)^{1 / 2} \\
& \leq 3\left(\left\|v_{+}(T)\right\|_{2}+\int_{0}^{T}\left\|F_{+}(t)\right\|_{2} d t\right) e^{4 \int_{0}^{T} c_{a, \beta}(\tau) d \tau} .
\end{aligned}
$$

To establish the first part of Theorem 1.1, the existence and uniqueness of a solution of (2.1), we apply the contraction principle in the space $X_{T}$ (2.6) with $\left(v_{+}^{m}, v_{-}^{m}\right)$ for $m \in \mathbb{N}$ the iteratively defined solution of the system

$$
\left\{\begin{array}{l}
\partial_{t} v_{+}^{m}=P_{+}\left(i \partial_{x}\left(a \partial_{x} v_{+}^{m}\right)-2 i a \phi \partial_{x} v_{+}^{m}\right)+\Lambda_{+}\left(v_{+}^{m-1}, v_{-}^{m-1}\right), \\
\partial_{t} v_{-}^{m}=P_{-}\left(i \partial_{x}\left(a \partial_{x} v_{-}^{m}\right)-2 i a \phi \partial_{x} v_{-}^{m}\right)+\Lambda_{-}\left(v_{+}^{m-1}, v_{-}^{m-1}\right), \\
v_{+}^{m}(x, T)=g(x) \in P_{+} L^{2}, \quad v_{-}^{m}(x, 0)=f(x) \in P_{-} L^{2}
\end{array}\right.
$$


where $v_{+}^{0}=v_{-}^{0}:=0$. The above equations are of the form (2.7) and (2.8), and the existence of solutions $v_{ \pm}^{m+1} \in C\left([0, T]: L^{2}(\mathbb{R})\right)$ will be proven below. Letting $\|v(t)\|_{2}:=\left\|v_{+}(t)\right\|_{2}+\left\|v_{-}(t)\right\|_{2}$, we have, from the energy estimates (2.9) and (2.10),

$$
\sup _{t \in[0, T]}\left\|v^{m+1}(t)\right\|_{2} \leq 3\left(\delta+2 \sup _{t \in[0, T]}\left\|v^{m}(t)\right\|_{2} \int_{0}^{T} K(t) d t\right) e^{4 \int_{0}^{T} c_{a, \beta}(\tau) d \tau}
$$

for $m \in \mathbb{N}$. From our choice of $T$ in (2.5) $), \sup _{t \in[0, T]}\left\|v^{1}(t)\right\|_{2} \leq 3 \delta e^{4 \int_{0}^{T} c_{a, \beta}} \leq 6 \delta$, and if we assume $\sup _{t \in[0, T]}\left\|v^{m}(t)\right\|_{2} \leq 8 \delta$, then the energy estimate (2.12) yields

$$
\sup _{t \in[0, T]}\left\|v^{m+1}(t)\right\|_{2} \leq 3(\delta+2(8 \delta)(1 / 48)) 2 \leq 8 \delta .
$$

Repeating the derivation of the energy estimates for the equations for the differences $v_{+}^{m+1}-v_{+}^{m}$ and $v_{-}^{m+1}-v_{-}^{m}$ and using (2.5) yields the estimate

$$
\sup _{t \in[0, T]}\left\|\left(v^{m+1}-v^{m}\right)(t)\right\|_{2} \leq \frac{1}{2} \sup _{t \in[0, T]}\left\|\left(v^{m}-v^{m-1}\right)(t)\right\|_{2} .
$$

By the contraction principle, there exists a unique solution

$$
\left(v_{+}, v_{-}\right) \in C\left([0, T]: L^{2}(\mathbb{R}) \times L^{2}(\mathbb{R})\right)
$$

of the system (2.3) (which is realized in $C\left([0, T]: H^{-2}(\mathbb{R})\right)$ ) with data $v_{+}(x, T)=$ $g(x) \in P_{+} L^{2}$ and $v_{-}(x, 0)=f(x) \in P_{-} L^{2}$.

To complete the above argument, we shall use the artificial viscosity method to prove the existence of solutions of (2.8) with initial data specified at 0 (similarly, one can prove the existence of solutions to (2.7) with data specified at time $T$ ). Thus, we consider the family of equations

$$
\begin{aligned}
\partial_{t} v_{-}^{\epsilon} & =-P_{-}\left(\epsilon \partial_{x}^{4} v_{-}^{\epsilon}-i \partial_{x}\left(a \partial_{x} v_{-}^{\epsilon}\right)+2 i a \phi \partial_{x} v_{-}^{\epsilon}\right)+F_{-} \\
& =-\epsilon P_{-} \partial_{x}^{4} v_{-}^{\epsilon}+\Phi\left(v_{-}^{\epsilon}\right), \quad v_{-}^{\epsilon}(\cdot, 0)=f(x) \in P_{-} L^{2}, \quad t>0 .
\end{aligned}
$$

By Duhamel's principle and the fact that the data is in $P_{-} L^{2}$, the solution $v_{-}^{\epsilon}(t)$ satisfies

$$
v_{-}^{\epsilon}(t)=e^{-\epsilon t \partial_{x}^{4}} v_{-}(0)+\int_{0}^{t} e^{-\epsilon\left(t-t^{\prime}\right) \partial_{x}^{4}} \Phi\left(v_{-}^{\epsilon}\left(t^{\prime}\right)\right) d t^{\prime} .
$$

From the inequality

$$
\left\|\partial_{x}^{j} e^{-\epsilon t \partial_{x}^{4}} f\right\|_{2} \leq c_{j}(\epsilon t)^{-j / 4}\|f\|_{2}, \quad j=0,1,2,3,
$$

it follows that

$$
\begin{aligned}
& \left\|v_{-}^{\epsilon}(t)\right\|_{2} \leq\left\|v_{-}(0)\right\|_{2} \\
& +\int_{0}^{t}\left\|e^{-\epsilon\left(t-t^{\prime}\right) \partial_{x}^{4}}\left\{\partial_{x}^{2}\left(a v_{-}^{\epsilon}\right)-\partial_{x}\left(\partial_{x} a v_{-}^{\epsilon}+2 a \phi v_{-}^{\epsilon}\right)+\left(2 \partial_{x}(a \phi) v_{-}^{\epsilon}+F_{-}\right)\right\}\right\|_{2} d t^{\prime} \\
& \leq\left\|v_{-}(0)\right\|_{2}+c \int_{0}^{t}\left\{\left(\frac{1}{\left(\epsilon\left(t-t^{\prime}\right)\right)^{1 / 2}}+\frac{1}{\left(\epsilon\left(t-t^{\prime}\right)\right)^{1 / 4}}+1\right)\left\|v_{-}^{\epsilon}\left(t^{\prime}\right)\right\|_{2}+\left\|F_{-}\right\|_{2}\right\} d t^{\prime} \\
& \leq\left\|v_{-}(0)\right\|_{2}+c\left(\frac{T^{1 / 2}}{\epsilon^{1 / 2}}+\frac{T^{3 / 4}}{\epsilon^{1 / 4}}+T\right) \sup _{t \in[0, T]}\left\|v_{-}^{\epsilon}(t)\right\|_{2}+\int_{0}^{T}\left\|F_{-}\right\|_{2} d t .
\end{aligned}
$$

A standard argument then shows the existence of a solution

$$
v_{-}^{\epsilon} \in C\left(\left[0, T_{\epsilon}\right]: L^{2}(\mathbb{R})\right)
$$


to (2.13), with $T_{\epsilon} \downarrow 0$ as $\epsilon \downarrow 0$. Using the a priori estimate (2.9), which holds uniformly in $\epsilon>0$, we reapply the above local argument to extend the solution $v_{-}^{\epsilon}$ to the time interval $[0, T]$, with $T$ as in (2.5), for all $\epsilon \in(0,1)$. Letting $\epsilon \rightarrow 0$ in an appropriate manner, we find the desired solution.

Since $v(x, t)=\varphi(x) u(x, t)$, both $u$ and $e^{\beta x} u$ are in $C\left([0, T]: L^{2}(\mathbb{R})\right)$, with $u$ solving (2.1) in $C\left([0, T]: H^{-2}(\mathbb{R})\right)$. Also, notice that

$$
w(x, t):=e^{\beta x} u(x, t) \in C\left([0, T]: L^{2}(\mathbb{R})\right)
$$

is a solution of the equation

$$
\begin{aligned}
\partial_{t} w & =i\left(\left(\partial_{x}-\beta\right) a\left(\partial_{x}-\beta\right) w+W(x, t) w(x, t)\right) \\
& =i \partial_{x}\left(a \partial_{x} w\right)-2 i \beta a \partial_{x} w+i\left(\beta^{2} a-\beta \partial_{x} a\right) w+i W w,
\end{aligned}
$$

with $w_{-}(x, 0)=P_{-}\left(e^{\beta x} u(x, 0)\right)$ and $w_{+}(x, T)=P_{+}\left(e^{\beta x} u(x, T)\right)$.

To prove the second part of Theorem 1.1. we project the above equation onto the positive and negative frequencies, obtaining a coupled system for $w_{ \pm}:=P_{ \pm} w$, from which we find the energy estimate

$$
\beta \int_{0}^{T} \int a(x, t)\left(\left|D_{x}^{1 / 2} w_{+}\right|^{2}+\left|D_{x}^{1 / 2} w_{-}\right|^{2}\right) d x d t \leq c\left(\left\|w_{-}(0)\right\|_{2}^{2}+\left\|w_{+}(T)\right\|_{2}^{2}\right)
$$

Therefore, from the hypothesis $a \geq \lambda>0$, we see that $w \in L^{2}\left([0, T]: H^{1 / 2}(\mathbb{R})\right)$.

We observe that formally $z(x, t)=D_{x}^{1 / 2} w(x, t)$ satisfies the equation

$$
\partial_{t} z=i \partial_{x}\left(a \partial_{x} z\right)-2 i \beta a \partial_{x} z+i \partial_{x}\left[D_{x}^{1 / 2} ; a\right] \partial_{x} w-2 i \beta\left[D_{x}^{1 / 2} ; a\right] \partial_{x} w+\Gamma(z, w),
$$

where $\Gamma(z, w)$ denotes a linear operator of "order zero" in $(z, w)$. Applying the projection operators, we obtain

$$
\begin{aligned}
\partial_{t} z_{ \pm}= & i \partial_{x}\left(a \partial_{x} z_{ \pm}\right)-2 i \beta a \partial_{x} z_{ \pm}+i \partial_{x}\left[P_{ \pm} ; a\right] \partial_{x} z-2 i \beta\left[P_{ \pm} ; a\right] \partial_{x} z \\
& +P_{ \pm}\left(i \partial_{x}\left[D_{x}^{1 / 2} ; a\right] \partial_{x} w-2 i \beta\left[D_{x}^{1 / 2} ; a\right] \partial_{x} w+\Gamma(z, w)\right) .
\end{aligned}
$$

Noticing that $\partial_{x}=D_{x}^{1 / 2} H D_{x}^{1 / 2}$, where $H$ is the Hilbert transform $(\widehat{H f}(\xi):=$ $i \operatorname{sgn}(\xi) \widehat{f}(\xi))$, and using Proposition 3.2, it follows that both

$$
\begin{aligned}
&\left|\int\left(P_{ \pm}\left[D_{x}^{1 / 2} ; a\right] \partial_{x} w\right) \overline{z_{ \pm}} d x\right|=\left|\int\left(\left[D_{x}^{1 / 2} ; a\right] D_{x}^{1 / 2} H z\right) \overline{P_{ \pm} z_{ \pm}} d x\right| \\
& \leq \leq\left\|J^{\delta} \partial_{x} a\right\|_{q}\|z\|_{2}\left\|z_{ \pm}\right\|_{2} \\
&\left|\int\left(P_{ \pm}\left(\partial_{x}\left[D_{x}^{1 / 2} ; a\right] \partial_{x} w\right)\right) \overline{z_{ \pm}} d x\right|=\left|\int\left(D_{x}^{1 / 2}\left[D_{x}^{1 / 2} ; a\right] D_{x}^{1 / 2} H z\right) \overline{D_{x}^{1 / 2} H z_{ \pm}} d x\right| \\
& \leq c\left\|J^{\delta} \partial_{x} a\right\|_{q}\left\|D_{x}^{1 / 2} z\right\|_{2}\left\|D_{x}^{1 / 2} z_{ \pm}\right\|_{2}
\end{aligned}
$$

hold, where we take $0<\delta<1,1<q<\infty$ such that $\delta>1 / q$. Since we know that $\|z\|_{L_{t}^{2} L_{x}^{2}}=\left\|D_{x}^{1 / 2} w\right\|_{L_{t}^{2} L_{x}^{2}} \leq c_{o}\left(c_{o}\right.$ denoting a constant depending on $\left\|w_{-}(0)\right\|_{2}$ and $\left.\left\|w_{+}(T)\right\|_{2}\right)$, we have that $\|z(t)\|_{L_{x}^{2}}<\infty$ for a.e. $t$. Therefore, for every $\epsilon>0$, we can find $t_{0}^{\epsilon} \in(0, \epsilon)$ and $t_{1}^{\epsilon} \in(T-\epsilon, T)$ such that $\left\|z\left(t_{i}^{\epsilon}\right)\right\|_{L_{x}^{2}} \leq c_{o}(\epsilon)$ for $i=0,1$. From equation (2.16), we obtain the following energy estimate for $z$ :

$$
\begin{aligned}
\beta \lambda \int_{t_{0}^{\epsilon}}^{t_{1}^{\epsilon}} \int\left|D_{x}^{1 / 2} z\right|^{2} d x d t & \leq \beta \int_{t_{0}^{\epsilon}}^{t_{1}^{\epsilon}} \int a(x, t)\left(\left|D_{x}^{1 / 2} z_{+}\right|^{2}+\left|D_{x}^{1 / 2} z_{-}\right|^{2}\right) d x d t \\
& \leq c_{o}(\epsilon)+c\left\|J^{\delta} \partial_{x} a\right\|_{L_{t}^{\infty} L_{x}^{q}} \int_{t_{0}^{\epsilon}}^{t_{1}^{\epsilon}}\left\|D_{x}^{1 / 2} z\right\|_{2}^{2} d t .
\end{aligned}
$$


By the hypothesis on the size of $\beta \lambda$, we can absorb the term on the right-hand side that arose from (2.18) into the left-hand side. This allows us to conclude that

$$
w \in C\left((0, T): H^{1 / 2}(\mathbb{R})\right), \quad D_{x} w \in L^{2}\left(\mathbb{R} \times\left[t_{0}^{\epsilon}, t_{1}^{\epsilon}\right]\right) \text { for every } \epsilon>0 .
$$

Reapplying this argument, it follows that $w=e^{\beta x} u \in C^{\infty}(\mathbb{R} \times(0, T))$.

\section{Appendix}

Lemma 3.1. Let $T$ denote one of the following operators: $P_{+}, P_{-}$, or $H$, the Hilbert transform. Then for any $p \in(1, \infty)$ and any $l, m \in \mathbb{Z}^{+} \cup\{0\}$ there exists $c=c(p ; l ; m)>0$ such that

$$
\left\|\partial_{x}^{l}[T ; a] \partial_{x}^{m} f\right\|_{p} \leq c\left\|\partial_{x}^{l+m} a\right\|_{\infty}\|f\|_{p}
$$

Proof. Without loss of generality we take $T=P_{+}$and observe that

$$
\partial_{x}^{l}\left[P_{+} ; a\right] h=\sum_{j=0}^{l} c_{j, l}\left[P_{+} ; \partial_{x}^{j} a\right] \partial_{x}^{l-j} h,
$$

for appropriate constants $c_{j, l}$, so it suffices to prove (3.1) with $l=0$. Also since

$$
\begin{array}{r}
{\left[P_{+} ; a\right] \partial_{x}^{m} f=P_{+}\left(a \partial_{x}^{m} f\right)-a P_{+} \partial_{x}^{m} f=P_{+}\left(a P_{-} \partial_{x}^{m} f\right)+P_{+}\left(a P_{+} \partial_{x}^{m} f\right)-a P_{+} \partial_{x}^{m} f} \\
=P_{+}\left(a P_{-} \partial_{x}^{m} f\right)-\left(I-P_{+}\right)\left(a P_{+} \partial_{x}^{m} f\right)=P_{+}\left(a P_{-} \partial_{x}^{m} f\right)-P_{-}\left(a P_{+} \partial_{x}^{m} f\right),
\end{array}
$$

it suffices to show the inequality

$$
\left\|P_{+}\left(a P_{-} \partial_{x}^{m} f\right)\right\|_{p} \leq c\left\|\partial_{x}^{m} a\right\|_{\infty}\|f\|_{p}
$$

and the corresponding inequality for $P_{-}\left(a P_{+} \partial_{x}^{m} f\right)$, the proof of which we omit since it is similar to the proof of (3.2). As we commented earlier, an inequality related to that in (3.2) was proved in [6].

To establish (3.2), we will use the Littlewood-Paley decomposition, following the approach and the notation given in [6]. First, we define functions $\eta$ and $\widetilde{\eta}$ centered at the frequencies \pm 1 . Let $\eta \in C_{0}^{\infty}(\mathbb{R}), \eta \geq 0$, supp $\eta \subseteq \pm(1 / 2,2)$ with the condition $\sum_{-\infty}^{\infty} \eta\left(2^{-k} \xi\right)=1$ for $\xi \neq 0$. Let $\widetilde{\eta} \in C_{0}^{\infty}(\mathbb{R}), \quad \widetilde{\eta} \geq 0$, supp $\widetilde{\eta} \subseteq \pm(1 / 8,8)$ with $\widetilde{\eta}(\xi)=1$ for $\xi \in \pm[1 / 4,4]$. Then, define the associated multiplication operators $Q_{k}$ and $\widetilde{Q}_{k}$ as follows: $\left(Q_{k} f\right)^{\wedge}(\xi):=\eta\left(2^{-k} \xi\right) \hat{f}(\xi)$ and $\left(\widetilde{Q}_{k} f\right)^{\wedge}(\xi):=\widetilde{\eta}\left(2^{-k} \xi\right) \hat{f}(\xi)$.

Let $P_{k} f:=\sum_{j \leq k-3} Q_{j} f$; therefore, $\left(P_{k} f\right)^{\wedge}(\xi)=p\left(2^{-k} \xi\right) \hat{f}(\xi)$ with $p(0)=1$ and supp $p \subseteq(-1 / 4,1 / 4)$. Finally, define the cutoff function $\tilde{p} \in C_{0}^{\infty}(\mathbb{R})$ with $\tilde{p}(\xi)=1$ for $\xi \in[-10,10]$ and let $\left(\widetilde{P}_{k} f\right)^{\wedge}(\xi)=\tilde{p}\left(2^{-k} \xi\right) \hat{f}(\xi)$.

Using the fact that $\left(Q_{k} f\right)^{\wedge}$ is supported on $\pm\left(2^{k-1}, 2^{k+1}\right)$ and $\left(P_{k} f\right)^{\wedge}$ is supported on $\left(-2^{k-2}, 2^{k-2}\right)$, we can compute that supp $\left(Q_{k} f P_{k} g\right)^{\wedge} \subseteq \pm\left(2^{k-2}, 2^{k+2}\right)$; therefore,

$$
Q_{k} f P_{k} g=\widetilde{Q}_{k}\left(Q_{k} f P_{k} g\right) .
$$

Also, since $\widetilde{P}_{k} f=f$ if supp $\hat{f} \subset\left(-10 \cdot 2^{k}, 10 \cdot 2^{k}\right)$, we see that for $|j| \leq 2$,

$$
Q_{k} f Q_{k-j} g=\widetilde{P}_{k}\left(Q_{k} f Q_{k-j} g\right) .
$$


To prove the needed estimate (3.2), we first take the dyadic decomposition of the functions on the left-hand side and split the double sum into three parts $(l-k \leq-3$, $l-k \geq 3$, and $|l-k| \leq 2)$ :

$$
\begin{aligned}
& P_{+}\left(a P_{-} \partial_{x}^{m} f\right)=P_{+}\left(\sum_{k, l} Q_{k} a P_{-}\left(Q_{l} \partial_{x}^{m} f\right)\right)=P_{+}\left(\sum_{k} Q_{k} a P_{-}\left(P_{k} \partial_{x}^{m} f\right)\right) \\
& +P_{+}\left(\sum_{k} P_{k} a P_{-}\left(Q_{k} \partial_{x}^{m} f\right)\right)+P_{+}\left(\sum_{|j| \leq 2} \sum_{k} Q_{k} a P_{-}\left(Q_{k-j} \partial_{x}^{m} f\right)\right)=: I+I I+I I I .
\end{aligned}
$$

Since for all $k \in \mathbb{Z}$, supp $\left(P_{k} a Q_{k}\left(P_{-} \partial_{x}^{m} f\right)\right)^{\wedge} \subset(-\infty, 0)$, it follows that $I I=0$. To estimate $I$, we use (3.3) to write

$$
\begin{aligned}
I & =\sum_{k} P_{+}\left(Q_{k} a P_{k}\left(P_{-} \partial_{x}^{m} f\right)\right)=\sum_{k} \tilde{Q}_{k}^{+}\left(Q_{k} a P_{k}\left(P_{-} \partial_{x}^{m} f\right)\right) \\
& =c \sum_{k} \iint e^{i x(\xi+\mu)} \tilde{\eta}^{+}\left(2^{-k}(\xi+\mu)\right) \eta\left(2^{-k} \xi\right) p\left(2^{-k} \mu\right) \mu^{m} \hat{a}(\xi) \chi_{\mathbb{R}^{-}}(\mu) \hat{f}(\mu) d \xi d \mu \\
& =c \sum_{k} \iint e^{i x(\xi+\mu)} m_{k}(\xi, \mu) \widehat{\partial_{x}^{m} a}(\xi)\left(\chi_{\mathbb{R}^{-}}(\mu) \hat{f}(\mu)\right) d \xi d \mu,
\end{aligned}
$$

where $m_{k}(\xi, \mu):=m\left(2^{-k} \xi, 2^{-k} \mu\right)$ and $m(\xi, \mu):=\tilde{\eta}^{+}(\xi+\mu) \eta(\xi) p(\mu)\left(\frac{\mu}{\xi}\right)^{m}$.

Let $q, h \in C_{0}^{\infty}(\mathbb{R})$ with $q \equiv 1$ on supp $\eta, h \equiv 1$ on supp $p$, supp $h \subset(-1 / 2,1 / 2)$, and supp $q \subset \pm(1 / 4,4)$, so that $m(\xi, \mu)=\tilde{\eta}^{+}(\xi+\mu) \eta(\xi) \mu p(\mu) \tau(\xi, \mu)$, with $\tau(\xi, \mu):=q(\xi) h(\mu) \mu^{m-1} / \xi^{m} \in C_{0}^{\infty}\left(\mathbb{R}^{2}\right)$. Thus, we can write the function $\tau$ as the Fourier transform of a Schwartz function:

$$
\tau(\xi, \mu)=c \iint e^{i(\xi \theta+\mu \nu)} r(\theta, \nu) d \theta d \nu, \quad \text { for some } \quad r \in \mathbb{S}\left(\mathbb{R}^{2}\right) .
$$

Hence,

$$
I=\int_{\nu} \int_{\theta} \sum_{k} \tilde{Q}_{k}\left(Q_{k}^{\theta}\left(\partial_{x}^{m} a\right) P_{k}^{\nu}\left(P_{-} f\right)\right) r(\theta, \nu) d \theta d \nu
$$

where the symbols of $Q_{k}^{\theta}$ and $P_{k}^{\nu}$ are $e^{i \theta 2^{-k} \xi} \eta\left(2^{-k} \xi\right)$ and $e^{i \nu 2^{-k} \mu} 2^{-k} \mu p\left(2^{-k} \mu\right)$, respectively, which belong to the class considered in [6] (page 607). So using Lemma A.3 in [6] and the Hardy-Littlewood maximal function $M$, it follows that

$$
\begin{aligned}
\left\|\sum_{k} \tilde{Q}_{k}\left(Q_{k}^{\theta}\left(\partial_{x}^{m} a\right) P_{k}^{\nu}\left(P_{-} f\right)\right)\right\|_{p} \leq c\left\|\left(\sum_{k}\left|Q_{k}^{\theta}\left(\partial_{x}^{m} a\right) P_{k}^{\nu}\left(P_{-} f\right)\right|^{2}\right)^{1 / 2}\right\|_{p} \\
\leq c\left\|\sup _{k}\left|Q_{k}^{\theta}\left(\partial_{x}^{m} a\right)\right|\left(\sum_{k}\left|P_{k}^{\nu}\left(P_{-} f\right)\right|^{2}\right)^{1 / 2}\right\|_{p} \\
\leq c\left\|M\left(\partial_{x}^{m} a\right)\right\|_{\infty}\left\|\left(\sum_{k}\left|P_{k}^{\nu}\left(P_{-} f\right)\right|^{2}\right)^{1 / 2}\right\|_{p} \leq c\left\|\partial_{x}^{m} a\right\|_{\infty}\|f\|_{p} .
\end{aligned}
$$

Finally, note that $I I I=0$ if $j=-2,-1$, or 0 . Then, using (3.4), we find that

$$
I I I=P_{+}\left(\sum_{j=1}^{2} \sum_{k} Q_{k}(a) Q_{k-j}\left(P_{-} \partial_{x}^{m} f\right)\right)=\sum_{j=1}^{2} \sum_{k} \tilde{P}_{k}^{+}\left(Q_{k}^{*}\left(\partial_{x}^{m} a\right) Q_{k-j}^{* *}\left(P_{-} f\right)\right),
$$

where the operators $Q_{k}^{*}$ and $Q_{k-j}^{* *}$ for $j=1,2$ are given by

$$
\widehat{Q_{k}^{*} h}(\xi):=\frac{\eta\left(2^{-k} \xi\right)}{\left(2^{-k} \xi\right)^{m}} \hat{h}(\xi), \quad \widehat{Q_{k-j}^{* *} h}(\xi):=\left(2^{-k} \xi\right)^{m} \eta\left(2^{-(k-j)} \xi\right) \hat{h}(\xi) .
$$


The symbols of these multipliers lie in the class considered in [6] and $\tilde{P}_{k}$ is uniformly bounded in $L^{p}$, so an argument similar to (3.5) provides the desired inequality.

Proposition 3.2. Let $\alpha \in[0,1), \beta \in(0,1)$ with $\alpha+\beta \in[0,1]$. Then for any $p, q \in(1, \infty)$ and for any $\delta>1 / q$ there exists $c=c(\alpha ; \beta ; p ; q ; \delta)>0$ such that

$$
\left\|D_{x}^{\alpha}\left[D_{x}^{\beta} ; a\right] D_{x}^{1-(\alpha+\beta)} f\right\|_{p} \leq\left\|J^{\delta} \partial_{x} a\right\|_{q}\|f\|_{p},
$$

where $J:=\left(1-\partial_{x}^{2}\right)^{1 / 2}$.

Note. The inequality (3.6) still holds with the same proof for $\tilde{D}_{x}^{s}=H D_{x}^{s}$ in place of $D_{x}^{s}$. Also, in the case $\beta=1$, we can use $\left[D_{x} ; a\right] f=[H ; a] \partial_{x} f+H\left(\partial_{x} a f\right)$ and (3.1) to obtain the inequality (3.6) with $q=\infty$ and $\delta=0$.

Proof. We observe that

$$
D_{x}^{\alpha}\left[D_{x}^{\beta} ; a\right] D_{x}^{1-(\alpha+\beta)} f=\left[D_{x}^{\alpha+\beta} ; a\right] D_{x}^{1-(\alpha+\beta)} f-\left[D_{x}^{\alpha} ; a\right] D_{x}^{1-\alpha} f .
$$

Therefore, it suffices to consider the case $\alpha=0$. But the proof of this case follows by combining the argument in Proposition A.2, Lemma A.3, and Theorem A.8 in the appendix of [6] with $\alpha=1$ and the Sobolev inequality, so it will be omitted.

\section{REFERENCES}

1. Calderón, A. P., Commutators of singular integral operators, Proc. Nat. Acad. Sci. U.S.A. 53 (1965), 1092-1099. MR0177312 (31:1575)

2. Craig, W., Kappeler, T., and Strauss, W., Microlocal dispersive smoothing for the Schrödinger equation, Comm. Pure Appl. Math. 48 (1995), 769-860. MR.1361016 (96m:35057)

3. Doi, S., Smoothing effects for Schrödinger evolution equation and global behavior of geodesic flow, Math. Ann. 318 (2000), 355-389. MR1795567 (2001h:58045)

4. Escauriaza, L., Kenig, C. E., Ponce, G., and Vega, L., On uniqueness properties of solutions of Schrödinger equations, Comm. PDE 31 (2006), 1811-1823. MR2273975

5. Kato, T., On the Cauchy problem for the (generalized) Korteweg-de Vries equation, Advances in Mathematics Supplementary Studies, Studies in Applied Math. 8 (1983), 93-128. MR759907 (86f:35160)

6. Kenig, C. E., Ponce, G., and Vega, L., Well-posedness and scattering results for the generalized Korteweg-de Vries equation via the contraction principle, Comm. Pure Appl. Math. 46 (1993), 527-620. MR 1211741 (94h:35229)

7. Kenig, C. E., Ponce, G., and Vega, L., On unique continuation for nonlinear Schrödinger equations, Comm. Pure Appl. Math. 60 (2002), 1247-1262. MR.1980854 (2004g:35195)

8. Mizohata, S., On the Cauchy problem, Notes and Reports in Math. in Science and Engineering, Science Press \& Academic Press 3 (1985). MR860041 (89a:35007)

9. Molinet, L., and Ribaud, F., Well-posedness results for the generalized Benjamin-Ono equation with arbitrary large initial data, Int. Math. Res. Notices 70 (2004), 3757-3795. MR2101982 (2005j:35200)

Department of Mathematics, University of Arizona, Tucson, Arizona 85721-0089

E-mail address: Idawson@math.arizona.edu

Department of Mathematics, University of California, Santa Barbara, California 93106

E-mail address: helena@math.ucsb.edu

Department of Mathematics, University of California, Santa Barbara, California 93106

E-mail address: ponce@math.ucsb.edu 